\title{
Estrategias didácticas para disminuir problemas de aprendizaje en población vulnerable
}

Ángela Yamile Torres Merchán* Heidy Mayerly Correa Valderrama*:

Artículo de reflexión

Fecha de Recepción: 3 noviembre 2017

Fecha de Aprobación: 30 marzo 2018

\section{Resumen}

Esta investigación tiene cómo propósito determinar estrategias didácticas tendientes a abordar las problemáticas de aprendizaje en población vulnerable de grado quinto de primaria del colegio Francisco de Paula Santander, utilizando la mediación para modificar las estructuras mentales de los sujetos, tal y como lo propuso Reuven Feuerstein, permitiendo que el docente actúe como mediador en el proceso de enseñanza aprendizaje, transformando en habilidades mentales las experiencias dejadas por el contexto.
El proyecto busca identificar problemas de aprendizaje en los estudiantes, elaborar estrategias didácticas acordes a la problemática, contando para esto con el apoyo de las nuevas tecnologías de la información y la comunicación (TIC), aplicarlas y evaluarlas teniendo como soporte el enfoque cualitativo y la Investigación Acción Educativa.

Palabras Clave: Mediación, estrategias didácticas, problemas de aprendizaje, aprendizaje, población vulnerable, educación.
* Colegio Técnico Municipal Francisco de Paula Santander - Boyacá Colombia

docenteangelatorres@ gmail.com

** Colegio Técnico

Municipal Francisco de Paula Santander - Boyacá Colombia maye_018@hotmail.com

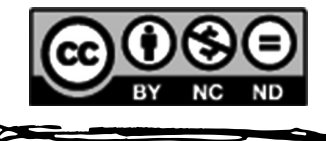




\section{Estrategias didácticas para disminuir problemas de aprendizaje en población vulnerable.}

\section{Introducción}

La educación moderna busca una escuela que genere los espacios apropiados para aprender, de manera integral $y$ formar seres humanos aptos para convivir en la sociedad, sin olvidar que cada sujeto es único, con habilidades y dificultades. Se busca que el sujeto cuente con las competencias que la sociedad exige para poder ser exitosos; por esta razón se busca que el docente sea generador de estrategias que se aplicaran en el aula, y al mismo tiempo sirva de mediador del aprendizaje, con el fin de mejorar los resultados académicos, pues para lograr aprendizajes el niño debe aprender a aprender con la ayuda de un docente mediador efectivo con las capacidades necesarias para mover, transformar y modificar las estructuras mentales de los estudiantes, incluso de aquellos que por determinadas situaciones ambientales, sociales u orgánicas tienen dificultad en el aprendizaje y comprensión de conceptos.

En el Colegio Técnico Municipal Francisco de Paula Santander de la ciudad de Duitama se ha podido evidenciar una situación problema en el aprendizaje de los niños y niñas de la institución, manifestado en los bajos resultados académicos y los resultados de pruebas internas y externas, debido a las condiciones de vulnerabilidad que presenta la población, entre ellas: La pobreza, maltratos, abusos, la falta de alguno de sus padres, las secuelas que ha dejado la violencia, la presencia de alguna discapacidad física o cognitiva, y cada una de estas atañen a los estudiantes de la institución.

Los niños y niñas del colegio Francisco de Paula Santander son hijos de vendedores ambulantes, empleados de talleres, minoristas de la plaza de mercado, constructores, empleadas domésticas, transportadores, recicladores, trabajadoras sexuales e incluso hijos de delincuentes y presidiarios; son niños y niñas desplazados por la violencia.

A través de las estrategias que se aplicarán en la investigación se pretende que el estudiante se adapte a su contexto y desarrolle habilidades para sobrellevar su diario vivir, que aprenda a aprender a pesar de las vivencias que le genera su contexto. Tzaban (2003) afirma que los seres humanos pueden cambiar para adaptarse a una problemática del medio ambiente, a esto se le llama aprender; tomando esto como referencia se pretende primero que todo identificar y comprender los problemas de aprendizaje ocasionados por las vivencias y el contexto de los estudiantes objeto de estudio, luego, al realizar el análisis adecuado del contexto, la problemática y las posibles consecuencias que estos aspectos inciden en el estudiante y su capacidad de aprender, se concibe que el docente sea el mediador entre unos estímulos o estrategias didácticas, el estudiante y su aprendizaje, lo cual 
puede marcar la diferencia en la educación actual; cabe indicar que la investigación no pretende cambiar ni el ambiente familiar de los sujetos ni el contexto, pero si permitir al niño un mejor y más placentero desarrollo como estudiante, como sujeto pensante. La institución se ha caracterizado por la variedad de desafíos que yacen en la diversidad de la población que hace parte de la comunidad educativa; sin dejar de lado que la problemática evidenciada en el diagnóstico preliminar también es una problemática nacional, teniendo en cuenta que estamos en una etapa de conflicto armado y violencia generalizada, familias disfuncionales y esto hace a nuestros niños vulnerables y con dificultades para aprender y desenvolverse en su contexto apropiadamente. Partiendo de esta premisa, se indagaron otras investigaciones nacionales $\mathrm{e}$ internacionales que brindan aportes conceptuales y experimentales que se encuentren relacionados con la investigación; en Caballero (2008), realiza un proyecto relacionado con la comprensión lectora de los textos argumentativos en los niños de poblaciones vulnerables escolarizados en quinto grado de educación básica primaria, evidenciando la importancia de la intervención didáctica en un grupo y cómo a partir de estas se pueden cambiar las estructuras cognitivas en los estudiantes teniendo como base un mediador, siendo este el principal objetivo del proyecto. Por otro lado, en el año Arce (2006), en su investigación muestra la implementación de una ruta de inclusión educativa bajo la estrategia de mediación de Feuerstein, este proyecto resulta de interés para la investigación a desarrollar, porque el sustento teórico utilizado ofrece herramientas conceptuales que la complementa, así como el hecho de estar respaldado en la teoría de la Modificabilidad Cognitiva de Feuerstein teniendo como eje central el docente como mediador. Así mismo, la investigación de Rivero y otros (2013), quienes realizaron un proyecto de investigación sobre Tecnologías educativas y estrategias didácticas, este proyecto aporta a esta investigación herramientas conceptuales sobre las TIC como estrategia didáctica, teniendo en cuenta los criterios de selección en el que se hace uso de estrategias didácticas que favorecen la práctica pedagógica a través del uso de las TIC que se tienen a disposición en el entorno como herramienta fundamental para favorecer el aprendizaje en los niños y niñas.

Dentro de las investigaciones internacionales en el año Ordoñez (2005) investigó acerca de la mediación de aprendizajes y los logros educativos del profesor de educación primaria. El aporte que hace el autor de esta tesis con respecto al docente mediador, brinda unas posibles herramientas y pautas para nuevas formas de aprendizaje que potencialicen los conocimientos de los estudiantes, lo que permite tomarlo como base para el desarrollo de este proyecto, junto con Aparcana (2015) quien en su tesis titulada efectos del programa de modificabilidad cognitiva $\mathrm{y}$ enriquecimiento instrumental en niños con necesidades educativas especiales; nuevamente se rescata
Dentro de las investigaciones internacionales en el año Ordoñez (2005) investigó acerca de la mediación de aprendizajes y los logros educativos del profesor de educación primaria. 


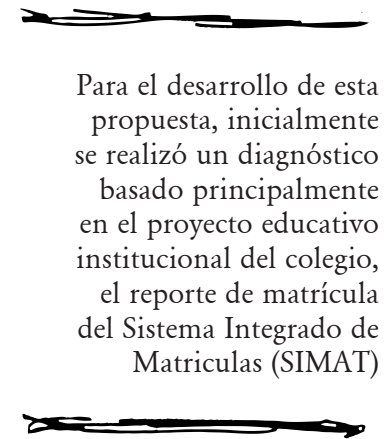

el hecho de que el programa de modificabilidad ya tiene antecedentes positivos en grupos de estudiantes que han sido comparados en pruebas, en relación con otros grupos, lo que aporta positiva y significativamente en el progreso de la investigación que se está desarrollando.

Cabe señalar que los estudios realizados soportan la investigación de manera positiva, dando aportes significativos en los conceptos teóricos y prácticos, además tener en cuenta las conclusiones de cada proyecto para así mejorar la investigación. Ahora bien, en esta investigación se ha decidido contar como estrategia principal con el apoyo de las nuevas tecnologías de la información y la comunicación (TIC), de estrategias didácticas encaminadas a permitir la creación de ambientes de aprendizaje donde la diversidad y las diferencias cobran importancia dando al docente un papel de mediador, con el fin de transformar no el contexto, sino la forma de afrontarlo y permitiendo que aquellas situaciones que hacen que esta población se encuentre en estado de vulnerabilidad y que afectan su capacidad de aprender, sean el detonante para el aprendizaje de los estudiantes. De esta manera las tecnologías de la información y la comunicación (TIC) como una de las estrategias pedagógicas y didácticas, permiten interacciones educativas entre estudiantes, que requiere recursos tecnológicos, físicos y humanos para enfocar positivamente y lograr el cambio en las aulas.
Para el desarrollo de esta propuesta, inicialmente se realizó un diagnóstico basado principalmente en el proyecto educativo institucional del colegio, el reporte de matrícula del Sistema Integrado de Matriculas (SIMAT), en donde se evidencia que la institución cuenta con niños con necesidades educativas especiales; también se elaboró una encuesta con el fin de identificar aspectos sociodemográficos de los niños de la institución; junto con la realización de entrevistas a los docentes, cómo herramienta de apoyo en el proceso de recolección de datos diagnósticos de identificación de problemas de aprendizaje y la claridad de si la institución cuenta realmente con población vulnerable, esto con el fin de tener un punto de partida acerca de las necesidades académicas y didácticas que se presentan en la institución y que se quieren mejorar con el diseño e implementación de una serie de estrategias didácticas, basadas en el uso de las TIC, como herramienta fundamental y en compañía del docente quien será el mediador en este proceso, tomando como base la teoría de Reuven Feuerstein. Finalmente, se evaluarán las estrategias didácticas implementadas con el fin de evidenciar la pertinencia de las mismas, y la disminución de los problemas de aprendizaje de los niños y las niñas de grado quinto.

En este sentido, surge cómo pregunta de investigación ¿Qué estrategias didácticas pueden ser aplicadas a población en condición de vulnerabilidad para disminuir problemas de aprendizaje del grado 
quinto del Colegio Técnico Municipal Francisco de Paula Santander?, la investigación será guiada por el modelo de investigación acción educativa, desde un enfoque cualitativo, ya que se considera que es la que se ajusta adecuadamente a los intereses de la investigación. Este concepto se aborda desde la perspectiva de Elliot (2000) quien afirma que la investigación acción es considerada como un camino para que los profesionales de la acción educativa comprendan la naturaleza de su práctica y puedan mejorarla a través de decisiones racionales nacidas del rigor de los análisis y no sólo de intuiciones, tanteos o arbitrariedades.

De acuerdo a esto y para lograr los objetivos de la investigación se han determinado algunas etapas, dentro de las cuales se encuentra inicialmente la etapa de diagnóstico de los problemas de aprendizaje que presentan los niños, basándose la investigación en diferentes documentos institucionales como las carpetas de la docente de apoyo, en donde hay el diagnóstico de la dificultad de aprendizaje que presentan los niños con necesidades educativas especiales; la información del SIMAT, una encuesta sociodemográfica, para determinar el contexto y las condiciones ambientales y familiares de los estudiantes, esto con el fin de corroborar la vulnerabilidad de la población, como paso a seguir se caracterizará para determinar la población vulnerable que se encuentra en el grado quinto, por último se realizará una entrevista a los docentes que orientan algún área en los grados cuartos y quintos y de acuerdo a los resultados provenientes de estos instrumentos de recolección de información se procederá a la creación de estrategias didácticas acordes a la edad, tipo de estudiantes y necesidades de aprendizaje, teniendo como base la teoría de Feuerstein, quien define al docente como mediador, junto con las actividades del programa de Enriquecimiento instrumental; el cual según Jara (2014) consiste en que el profesor en el aula pondrá en práctica estrategias de mediación en la presentación de las tareas, en la codificación, decodificación de los términos, construcción de los conceptos, preparación del trabajo independiente, en la exploración de los procesos, orientación espacial y en la construcción de "puentes" con otras áreas del conocimiento y de la vida en general, seguidamente se aplicaran dichas estrategias y serán evaluadas para comprobar su efectividad en el mejoramiento del desempeño escolar de los estudiantes (p 26).

En los siguientes apartes se dará a conocer el marco teórico, que sustenta el desarrollo de la investigación, así como la metodología y enfoque seleccionado, los cuales son los que se acomodan a las necesidades investigativas del proyecto, finalmente se dará a conocer los resultados esperados.

\section{Marco teórico}

\section{Modificabilidad estructural cognitiva}

La investigación parte de la idea de mejorar y hacer efectivo el aprendizaje de los estudiantes, para esto se 
toma como base la teoría de Reuven Feuerstein, acerca de la Modificabilidad Estructural cognitiva, partiendo del planteamiento realizado por Jean Piajet sobre estructuras mentales, quien afirma que el contacto con los objetos y las relaciones interpersonales modifican los esquemas mentales, para dar mayor claridad, cada vez que hay un estímulo adecuado, sea positivo o negativo, en el cerebro se modifican unas estructuras ocasionando determinados cambios en la forma de actuar, a lo que Piaget llama asimilación y acomodación, y es ahí cuando sucede el proceso de aprender. Fundamentándonos en esta teoría, aquellos niños que no tengan la capacidad de modificar sus estructuras cognitivas, o en otras palabras que tienen problemas de aprendizaje, los cuales han sido determinados por diferentes factores, ambientales o sociales, podrían obtener los aprendizajes con la ayuda de un mediador, modificándose y adaptándose a sus vivencias y además logrando aprender. Es por esta razón que se tiene la necesidad de buscar unas estrategias que respondan a las solicitudes que se presentan en el aula, tomando como base principal para mejorar el proceso de enseñanza aprendizaje la teoría de Feuerstein quien define el aprendizaje mediado como:

La forma en que los estímulos emitidos por el ambiente son transformados por un agente mediador (padre, madre, hermano, profesor u otro). El agente mediador, haciendo uso de sus intenciones, cultura y emociones, selecciona y organiza los estímulos más apropiados para el estudiante; de manera que en futuras situaciones este mismo logre identificar, clasificar y organizar los estímulos más relevantes que debe aprender en una situación en deterioro de otros menos importantes. "La mediación consiste en favorecer el desarrollo de los procesos y estrategias del pensamiento que no sólo están implícitos en las actividades escolares sino también en las situaciones de la vida social y familiar del niño"; (Orru, 2003, p. 37).

Con el fin de disminuir o erradicar los problemas de aprendizaje generados por las características que presenta la población vulnerable y que se detectaron durante el desarrollo de la investigación, se ha determinado la importancia de las estrategias didácticas como recurso para el mejoramiento del desempeño académico de los estudiantes, tomando las estrategias didácticas cómo herramientas de gran importancia para realizar modificaciones cognitivas en los niños y niñas, en otras palabras para lograr verdaderos aprendizajes, significativos, y que a hacer de los estudiantes personas útiles y competitivas en la sociedad donde nos encontramos para esto tomamos a Fonseca y Aguded, (2007) quienes afirman que "las estrategias didácticas utilizadas en el proceso de enseñanza aprendizaje de los estudiantes resultan necesarias en cualquier proceso formativo, las estrategias didácticas también son conocidas como estrategias para la mediación pedagógica, estrategias de enseñanza o formas de enseñanza”. 
Por esta razón y teniendo en cuenta la propuesta de investigación, las estrategias didácticas son acordes para el momento de implementación de la misma. De otro modo, los avances tecnológicos y las exigencias de la sociedad del conocimiento, coloca sobre los docentes la responsabilidad de pensar espacios educativos que fomenten el aprendizaje autónomo e integren conocimientos y competencias universitarias a la vez.

Lo que se quiere con las estrategias didácticas desarrolladas desde la Modificabilidad estructural cognitiva es que los estudiantes tengan la capacidad de transformar sus estructuras mentales, con el fin de desarrollar nuevas destrezas, así como estimular los dispositivos básicos de aprendizaje tales como atención percepción y memoria; esto sólo se logra según Feuerstein (1990) con la ayuda de un mediador que puede ser la escuela, el padre o madre de familia, un compañero o el mismo docente, afirmando que el aprendizaje mediado por otro ser humano es indispensable para un niño o una niña puesto que les ayuda a crear aquellos pre-requisitos cognitivos que luego hacen que el aprendizaje directo sea efectivo. (p. 39).

En este aspecto es importante resaltar que el agente mediador como lo menciona Feuerstein debe tener una fuerte conexión afectiva con el sujeto mediado, para que inicialmente surja una motivación dada por la afectividad, y así permitir que el estudiante desarrolle habilidades de pensamiento sin importar la clase de estímulos que sean brindados por el contexto. En virtud de lo indicado es importante recalcar que esta investigación no pretende cambiar el contexto en el que se desarrollan los niños y niñas de la institución, no se puede cambiar a sus familias ni las experiencias ya vividas o las que están por vivir, pero si se puede modificar sus estructuras mentales para que obtengan los aprendizajes necesarios para ser exitosos e inicialmente mejoren sus procesos de aprendizaje en los procesos educativos.

A su vez, desde esta nueva perspectiva del quehacer pedagógico, se debe transformar el rol del docente, buscando la innovación y elaboración de estrategias o herramientas que permitan el desarrollo del pensamiento de los estudiantes, basándose en situaciones problema con el fin de ejercitar operaciones mentales, conductas y habilidades que los haga sujetos más competentes, y ya que la teoría de Feuerstein habla del docente como mediador, no se trata sólo de la creación de estrategias, sino de las habilidades sociales para relacionarse con los estudiantes, y así lograr un pertinente aprendizaje mediado, de lo contrario si un docente no tiene la cercanía apropiada a sus estudiantes, podría crear variedad de estrategias y ninguna permitiría el desarrollo de procesos de pensamiento adecuado en sus estudiantes, ni las conexiones neuronales que se quiere lograr.

Aunado a lo anterior, la necesidad de buscar estrategias que disminuyan los problemas de aprendizaje es una 
La importancia de las TIC en la sociedad y en las prácticas pedagógicas (Muñoz, 2016) actuales lleva a analizarlas y tenerlas en cuenta en el desarrollo de la propuesta de investigación ya que cómo dice Fandos

(2002) urgencia educativa, y la sociedad actual está en cambios constantes cada vez a un mayor ritmo, eso sin contar con que la sociedad actual se encuentra en la era de la tecnología, donde los niños y jóvenes nacen con el gusto por las Tecnologías de la Información (TI) , por esta razón los estudiantes se han visto interesados por estas herramientas tecnológicas, partiendo de esto, los docentes deben buscar estrategias para que estos instrumento sean un material útil y no una problemática en el desarrollo adecuado de las clases, se evidencia el interés por los estudiantes en el uso de la Tecnología, lo que permitiría que sea una herramienta mediadora en el proceso enseñanza aprendizaje Fandos (2002).

La importancia de las TIC en la sociedad y en las prácticas pedagógicas (Muñoz, 2016) actuales lleva a analizarlas y tenerlas en cuenta en el desarrollo de la propuesta de investigación ya que cómo dice Fandos (2002), "La incorporación de las TIC en el proceso de mediación y la consecuente evolución del concepto de enseñanza, fruto a su vez de una transformación social (...). El potencial para la comunicación y acceso a la información de estas herramientas nos permite un 'valor añadido' a los procesos de enseñanza-aprendizaje que, en función de su adecuación didáctica, ofrecerá la posibilidad de mejorar estos procesos (...). El grado de innovación que ha significado el uso de las TIC en educación está en relación con la influencia que estos medios han tenido sobre los demás elementos que configuran el acto didáctico y que habría que analizar a través del comportamiento del modelo didáctico mediador” (p. 33)

Es así como con la ayuda de las estrategias nombradas anteriormente, y de acuerdo al tipo de investigación que se desarrolla, se seleccionaron los instrumentos para la recolección de información los cuales se encuentran íntimamente ligados a cada una de las etapas del proyecto.

\section{Metodología}

La investigación se plantea desde un enfoque de investigación cualitativo, el cual según De Souza (1997) es con el que se pretende interpretar lo que acontece en un fenómeno social; tal y como es el caso de la educación. Según Gonzales (1997)La investigación que se propone requiere de la investigación cualitativa para comprender los problemas de aprendizaje e intervenirlos mediante el quehacer docente, buscando transformar la realidad por medio de la relación enseñanza-aprendizaje, la cual se caracteriza por contar con un fuerte componente de interacción humana, aspecto que hace compleja y subjetiva la investigación, donde se vincula al objeto y al sujeto de investigación desde su cultura y sus formas de pensar, percibir y actuar en sus vivencias sociales; motivos que enmarcan la presente propuesta investigativa en el campo de la investigación cualitativa.

Por otro lado, Taylor (1998) afirma que con el enfoque cualitativo se busca describir la forma en que acontecen los fenómenos Tal descripción se hace 
desde la perspectiva del sujeto, desde su sentir y sus vivencias. En éste caso, las vivencias se encuentran asociadas a los estudiantes y los docentes, quienes asumen a diario el reto de desarrollar un proceso formativo en un escenario de vulnerabilidad.

Sandoval (2002) afirma que la investigación cualitativa requiere de puntos de referencia epistemológicos, ya que son los que le dan sentido a los momentos en los que se ha conceptualizado el proceso de investigación cualitativa. Los momentos en cuestión son los de formulación, diseño, gestión y cierre. Realizando un proceso humanista holístico, acorde con la investigación planteada.

La metodología de la investigación está basada en la investigación acción educativa puesto que es la que más se acomoda a las necesidades investigativas y sociales del proyecto ya que la investigación partió de la necesidad de mejorar el rendimiento académico de los estudiantes, acarreando diferentes problemáticas sociales, queriendo hacer una transformación social, y repensando la labor del docente como es la finalidad de la investigación acción. Para sustentar lo anterior Restrepo (2004) recuenta como la investigación-acción se orientó más a la transformación de prácticas sociales que a la generación o descubrimiento de conocimiento nuevo. Restrepo (2004) cita a Kurt Lewin, quien propone de esta metodología, las tres fases, que han subsistido El punto de partida es el diagnóstico acerca de la práctica social. Así como la primera etapa del proyecto en curso. La investigación acción educativa también propone una segunda fase que es quien direcciona el proyecto de investigación mencionado, la cual se encarga según Restrepo (2004) de reconstruir la práctica, la propuesta de una práctica alternativa más efectiva. Conocidas las falencias de la práctica anterior y presente, es posible incursionar en el diseño de una práctica nueva.

Finalmente, la tercera fase tiene que ver con la validación de la efectividad de la práctica alternativa o reconstruida, es decir, con la constatación de su capacidad práctica, para lograr bien los propósitos de la educación. La nueva práctica no debe convertirse en el nuevo discurso pedagógico sin una prueba de efectividad (Pág. ,51).

Y es así como las etapas del proyecto en mención coinciden acertadamente con las etapas propuestas en la investigación acción educativa, para contextualizar la primera etapa se tiene en cuenta que para la investigación el grupo control fue de 35 estudiantes de básica primaria. Estos estudiantes se encuentran en edades entre los 8 y los 12 años, en estratos socioeconómicos en 0,1 y 2 con un total de 17 niñas y 18 niños de los cuales una niña y un niño son afro descendientes; la mayoría de ellos viven en el área urbana en barrios de tolerancia estos datos han sido determinados de acuerdo a las encuestas sociodemográficas que se realizaron como parte del diagnóstico 
cumpliendo con la primera etapa de la investigación, también se evidenció que dentro de las entrevistas aplicadas a los estudiantes se corrobora que viven en barrios en donde presentan altos grados de delincuencia e inseguridad, justifican problemas de violencia intrafamiliar, de adicciones a algún tipo de sustancia, sin embargo se corrobora que a pesar de esto los estudiantes sienten agrado por el lugar donde habitan, son felices a pesar de algunas vivencias, les gusta su barrio.

Partiendo de las características de los sujetos objeto de la presente investigación, es importante resaltar y tener en cuenta el término de población vulnerable, el cual se acoge apropiadamente a las características de la población a investigar y debido a las particularidades socioculturales en que se encuentran los estudiantes, teniendo como base fundamental que si se logran aprendizajes efectivos con los niños y niñas en condición vulnerable, aún más se obtendrán logros a nivel de desempeño y competencia con aquellos niños $\mathrm{y}$ niñas que no presentan problemas de aprendizaje; para esto el Ministerio de Educación Nacional (2005) complementa teóricamente la investigación afirmando que: ya que hay diferentes factores que pueden dar origen a la desigualdad o pueden hacer a un sujeto vulnerable, el Ministerio de educación Nacional supone los siguientes aspectos: "aspectos económicos, históricos, culturales, políticos, económicos, físicos, sensoriales, emocionales o psicosociales" (MEN, 2005, p. 4).

Este concepto del Ministerio de Educación Nacional (MEN) complementa la afirmación de Feuerstein sobre población vulnerable y la importancia a nivel educativo que proporcionó el hecho que después de muchos estudios Feuerstein concluyó que la insuficiencia reducida de las personas para responder a sus fuentes de motivación y para modificar sus estructuras cognitivas, se debía a la carencia de mediación en los períodos decisivos de su desarrollo.

\section{Recolección de datos}

Las técnicas de análisis a empleadas dentro del proceso de investigación dependen del momento y el tipo de instrumento a emplear. Para la primera etapa que es la de diagnóstico se realizó una encuesta sociodemográfica, que a pesar de ser una técnica cuantitativa, no sustrae el enfoque cualitativo de la investigación; y una entrevista focal con el fin de identificar y corroborar el tipo de población a investigar, un análisis de texto a las entrevistas de estudiantes y docentes, con ello se pretende obtener una codificación de tipo formal y de esta manera poder identificar las subcategorías emergentes que se relacionan con los problemas de aprendizaje. Con los reportes de calificaciones, se elaborará un perfil de las asignaturas que presentan mayor dificultad para los estudiantes, a las que posteriormente se enfocará la implementación de las estrategias didácticas.

Las entrevistas a estudiantes y docentes de la segunda etapa son las mismas que se implementaron en la primera 
etapa y son insumo para determinar las estrategias didácticas acordes con las problemáticas identificadas. Para la tercera etapa, en los talleres grupales se realizarán anotaciones de los docentes sobre la dinámica que tienen las clases al ser implementadas las estrategias didácticas. Entretanto, las entrevistas grupales recogerán las percepciones de los estudiantes sobre la aplicación de las estrategias y se les aplicará un análisis textual semejante al de la primera etapa, pero para identificar fortalezas y oportunidades de mejora sobre las estrategias implementadas, esto con el fin de ser consecuentes con la investigación realizándose de manera holistica.

Finalmente, la cuarta etapa tomará los instrumentos de la tercera etapa y, los triangulará entre sí y con la teoría para establecer un juicio de valor asociado con la implementación de las estrategias, con la intención de mejorar las condiciones en las que se realiza la relación enseñanza aprendizaje, y desarrollar habilidades que le permitan a los estudiantes aprender a aprender.

\section{Técnicas de Análisis}

Para la etapa diagnóstica se realizó un análisis de texto a las entrevistas de estudiantes y docentes para obtener una codificación de tipo formal $y$ de esta manera poder identificar las subcategorías emergentes que se relacionan con los problemas de aprendizaje. Con los reportes de calificaciones, se elaborará un perfil de las asignaturas que presentan mayor dificultad para los estudiantes; asignaturas a las cuales se enfocará la implementación de las estrategias didácticas.

Para la tercera etapa, en los talleres grupales se realizarán anotaciones como diarios de campo de los docentes sobre la dinámica que tienen las clases al ser implementadas las estrategias didácticas. Entretanto, las entrevistas grupales recogerán las percepciones de los estudiantes sobre la aplicación de las estrategias y se les aplicará un análisis textual semejante al de la primera etapa, pero para identificar fortalezas y oportunidades de mejora sobre las estrategias implementadas.

Finalmente, los instrumentos de la tercera etapa serán triangulados entre sí y con la teoría para establecer un juicio de valor asociado con la implementación de las estrategias, con la intención de mejorar las condiciones en las que se realiza la relación enseñanza aprendizaje.

\section{Resultados preliminares}

De acuerdo a la entrevista focal aplicada a un grupo de 12 estudiantes, y a la encuesta sociodemográfica aplicada al grupo total de 35 estudiantes, se puede evidenciar lo siguiente:

Los niños son considerados como población vulnerable debido a las diferentes características ambientales, contextuales, familiares o sociales en las que viven, algunas de ellas son : violencia intrafamiliar, o vivir en un hogar disfuncional, pobreza extrema, son niños desplazados por la violencia,
Los niños son considerados como población vulnerable debido a las diferentes características ambientales, contextuales, familiares o sociales en las que viven, algunas de ellas son: violencia intrafamiliar, o vivir en un hogar disfuncional, pobreza extrema 


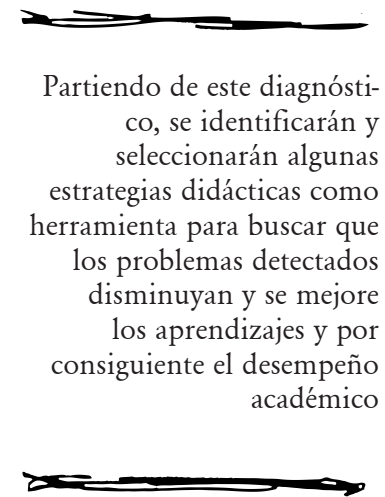

viven en barrios que presentan altos índices de delincuencia común, son niños maltratados, pero además de todo esto presentan algunos problemas de aprendizaje, que se considera que son generados por las diferentes condiciones de vulnerabilidad en la que se encuentran los niños, algunos de los problemas detectados en el diagnóstico inicial que es la etapa en la que se encuentra la investigación son: problemas de lenguaje, dislexia, disgráfia, necesidades educativas especiales (discapacidad intelectual, autismo), cabe resaltar que hay algunas condiciones que no han sido manifestadas directamente por los niños, pero que se puede inferir por las actitudes o información que detectan los docentes.

Partiendo de este diagnóstico, se identificarán y seleccionarán algunas estrategias didácticas como herramienta para buscar que los problemas detectados disminuyan y se mejore los aprendizajes y por consiguiente el desempeño académico

\section{Alcances y limitaciones}

El proyecto pretende que por medio de estrategias didácticas basadas en la modificabilidad estructural cognitiva se logre que la población vulnerable del grado 5 de primaria del colegio Técnico Municipal Francisco de Paula Santander, disminuya los problemas de aprendizaje determinados por la problemática social y cultural a la que se encuentran expuestos en su día a día; cambiando sus estructuras cognitivas para lograr en ellos sujetos más críticos con la capacidad de transformar su realidad y del mismo modo se espera que puedan resolver situaciones problema donde requieran retomen estos conceptos y estrategias y los aplique a su contexto.

Actualmente se está desarrollando el primer objetivo de la propuesta, realizar un diagnóstico de los problemas de aprendizaje de los niños de grado $5^{\circ}$ mediante la aplicación de entrevistas y encuestas de caracterización a docentes y estudiantes, teniendo en cuenta la premura del tiempo para el desarrollo de la investigación y de acuerdo a los resultados de las pruebas saber, ICSE, resultados académicos internos se definió trabajar solamente en el área de lectura, ya que es una de las que se ha venido presentando con alto porcentaje de desempeño mínimo y bajo.

La mayor de las limitaciones para el desarrollo del proyecto es la premura del tiempo para su desarrollo, así como los recursos económicos y tecnológicos, pues la institución cuenta con excelentes recursos pero el acceso a estos es restringido; al igual que la implementación de las estrategias didácticas a un solo grupo de la institución, en el cual se va a abordar las problemáticas específicas, delimitando la evaluación de estas a unos resultados parciales y a la disposición por aprender de los estudiantes de ese grupo específico.

\section{Impacto y resultados esperados}

Al finalizar con el proceso de investigación se espera contar con un grupo de estrategias didácticas que 
puedan ser implementadas en todos los grupos de estudiantes, para disminuir los problemas de aprendizaje de población vulnerable. Asimismo, con las estrategias didácticas obtenidas se busca impactar en la forma en que los estudiantes de poblaciones vulnerables asumen su proceso formativo, $\mathrm{y}$ asimilan los estímulos del medio en el que viven, ofreciéndoles una alternativa de crecimiento personal basada en el conocimiento. Igualmente se pretende con el desarrollo de una investigación humanista hacer de los estudiantes sujetos activos, capaces de transformar su realidad, apoyados en la mediación del docente.

\section{Referencias}

Aparcana, C. (2015). Efectos del programa de modificabilidad cognitiva y enriquecimiento instrumental en niños con necesidades educativas especiales (Tésis de maestría). Universidad Femenina Del Sagrado Corazón, Perú.

Bonilla, E. (1995). Más allá del dilema de los métodos. La investigación en ciencias sociales. Bogotá: Grupo editorial Norma.

Arce, V. (2016). Implementación de una ruta de inclusión educativa bajo la estrategia de mediación de Feuerstein (Tesis de maestría). UNAD, Eje Cafetero.

Caballero E. (2008). Comprensión lectora de los textos argumentativos en los niños de poblaciones vulnerables escolarizados en quinto grado educación básica primaria (Tesis de maestría). Universidad de Antioquia, Medellín.

Colombiaaprende. ¿Qué son poblaciones vulnerables? Recuperado de http://www. colombiaaprende.edu.co/html/mediateca/1607/article-85443.html

De Souza Minayo, M. (1997). El desafío del conocimiento. Buenos Aires: Lugar editorial.

Elliot J. (2000) La investigación-acción en educación. Ediciones Morata S.L. Cuarta Edición

Feuerstein. R. (1990). La Teoría de Modificabilidad Estructural Cognitiva [Pdf].

Fonseca, M. \& Aguded, I. (2007). Enseñar en la universidad. Experiencias y propuestas para la docencia universitaria. La Coruña: Netbiblio.

Jara L (2014) Programa de enriquecimiento instrumental para desarrollar las funciones cognitivas en el octavo año de educación básica de la unidad educativa salesiana maría auxiliadora.(Tesis de maestría). Universidad de cuenca. Cuenca Ecuador 
Martínez M. (2000). Investigación acción en el aula. Universidad Simón Bolívar. Agenda Académica, 7(1).

Muñoz, H. A. (2016). Mediaciones tecnológicas: nuevos escenarios de la práctica pedagógica. Praxis \& Saber, 7 (13), 199-221. http://dx.doi. org/10.19053/22160159.4172

Ordoñez Díaz, J. (2005). La mediación de aprendizajes y los logros educativos del profesor de educación primaria (Tesis de maestría). ITESM-Universidad Virtual, México.

Orru, S. E. (2003). Reuven feuerstein y la teoría de la modificabilidad cognitiva estructural. Revista de educación (332).

Restrepo, B. (2004) La investigación- acción educativa y la construcción de saber pedagógico. Revista Educación y Educadores, 7.

Rivero, I., Gómez, M. \& Abrego R. (2013). Tecnologías educativas y estrategias didácticas: criterios de selección. Revista Ciencia y Tecnología. (3), 190-206.

Rivero, I., Gómez, M. \& Abrego R. (2013). Tecnologías educativas y estrategias didácticas: criterios de selección. Revista Ciencia y Tecnología. (3), 190-206.

Tzaban, N. (2003):”Reuven Feuerstein y la Teorìa de la Modificabilidad Cognitiva Estructural [https://www.youtube.com/watch?v=xKtUZz2d-WM]. Recuperado de: Universidad Técnica Particular de Loja UNIDAD PARA EL DESARROLLO DEL PENSAMIENTO. Publicado 2009.

UNESCO A. S. (2006, mayo). La sociedad, sus necesidades educativas y las TIC. Las tecnologías de la información y la comunicación en la enseñanza. Orrú, S. (2003). Reuven Feuerstein y la teoría de la modificabilidad cognitiva estructural. Revista de educación. (332), pp. 33-54. Disponible en: https:// www.mecd.gob.es/dctm/revista-de educacion/articulosre332/re3320311443. pdf?documentId=0901e72b81256ae0 